\title{
A New Abietane and Two Dimeric Abietane Diterpenes from the Black Heartwood of Cryptomeria japonica
}

\author{
Shigenobu Arihara, * Akemi Umeyama, Shinya Bando, Shinji Imoto, Mikako Ono, Mayo Tani, and \\ Kazuko YoshiKawa
}

Faculty of Pharmaceutical Sciences, Tokushima Bunri University; Yamashiro-cho, Tokushima 770-8514, Japan.

Received October 3, 2003; accepted December 2, 2003

\begin{abstract}
Three new diterpenes, sugikurojins $\mathrm{A}-\mathrm{C}(1-3)$ were isolated from the black heartwood of Cryptomeria japonica. The structure of sugikurojin A was deduced to be 19-hydroxy-6,7-dehydroferruginol on the basis of extensive NMR experiments. Sugikurojin B was a dimer of 6,7-dihydroxyferruginol and 6,7-dehydroferruginol with a 6-O-11' linkage. Sugikurojin $\mathrm{C}$ was a dimeric ferruginol with $6-O-7^{\prime}$ and $7-O-6^{\prime}$ linkages. Also obtained in this investigation were the known compounds formosaninol (4), 15 sesquiterpenes (5-19), 16 diterpenes (2035), three phenylpropanoids (36-38), and a phenolic compound (39).
\end{abstract}

Key words Cryptomeria japonica; Taxodiaceae; black heartwood; abietane; sugikurojin

The Japanese cedar, Cryptomeria japonica D. Don. (Taxodiaceae) is a widely distributed conifer called sugi in Japanese. This wood is the most popular building material for Japanese housing. Although many cedar cores are reddish brown, about $20 \%$ are black, as a result of genetic factors, physical damage, infection of fungus, etc., and are called kurojin in Japanese. Chemical research on the terpene components of cedar cores is limited to the reddish brown ones. ${ }^{1-9)}$ This prompted us to examined the terpene constituents of black heartwood.

Shaved chips $(3.92 \mathrm{~kg})$ of black heartwood were exhaustively extracted with $\mathrm{MeOH}$ at room temperature for 4 weeks. The extract was partitioned between $\mathrm{H}_{2} \mathrm{O}$ and EtOAc. Repeated separation of the EtOAc-soluble portion by chromatography over ordinary-phase silica gel and reverse-phase silica gel furnished a novel abietane, sugikurojin A (1), dimeric abietanes, and sugikurojins B (2) and C (3), along with known compounds formosaninol (4), ${ }^{10)} \alpha$-muurolene $(5),{ }^{11)} \delta$-cadinene $(\mathbf{6}),{ }^{12)}$ calamenene $(7),{ }^{13,14)}$ T-cadinol $(\mathbf{8}),{ }^{15)}$ cubenol (9), ${ }^{15)}$ epi-cubenol (10), ${ }^{16,17)}$ cubebol (11), ${ }^{16,17)}$ epicubebol (12), ${ }^{18)}$ gleenol (13), ${ }^{19)} \beta$-elemol (14), ${ }^{20)}$ cryptomerione $(\mathbf{1 5}),{ }^{21} \quad 7(14), 10$-bisaboladien-1-ol-4-one $\quad(\mathbf{1 6}),{ }^{22)}$ 2,7(14),10-bisabolatrien-1-ol-4-one (17), ${ }^{23)}$ 7(14),10-bisaboladien-1,4-diol (18), ${ }^{24)}$ cryptomeridiol (19) ${ }^{25)}$ sandracopimaradiene (20), ${ }^{26)}$ sandaracopimarinal (21), ${ }^{27)}$ sandaracopimarinol (22), ${ }^{28)}$ sandaracopimaric acid (23), ${ }^{29)}$ 8[14],15-pi- maradiene-3-ol (24), ${ }^{30)}$ 18-nor-8[14],15-isopimaradien-4-ol (25) ${ }^{31)}$ sandaracopimarinol acetate (26), ${ }^{31)}$ 16-phyllocladanol $(\mathbf{2 7}),{ }^{32)}$ 7,13-abietadiene (28), ${ }^{33)}$ abietatriene (29), ${ }^{34)}$ ferruginol (30), ${ }^{35)}$ 6,7-dehydroferruginol $(\mathbf{3 1}),{ }^{36)}$ sugiol $(\mathbf{3 2}),{ }^{37)}$ hinokiol $(\mathbf{3 3}){ }^{37)}$ 19-hydroxyferruginol $(\mathbf{3 4})^{3)}$ 6,7-secoferruginol-6,7-dial (35), ${ }^{38)}$ sequirin $\mathrm{C}(\mathbf{3 6}),{ }^{39)}$ sugiresinol $(\mathbf{3 7}),{ }^{39)}$ agatharesinol (38), ${ }^{39)}$ and 4-hydroxybenzaldehyde (39). Compounds 4-38 were identified by comparison with published data. ${ }^{1} \mathrm{H}-{ }^{1} \mathrm{H}$ correlation spectroscopy $\left({ }^{1} \mathrm{H}-{ }^{1} \mathrm{H}\right.$ COSY $),{ }^{1} \mathrm{H}$-detected heteronuclear multiple-quantum coherence (HMQC), ${ }^{1} \mathrm{H}$-detected heteronuclear multiple-bond connectivity (HMBC), and rotating frame nuclear Overhauser and exchange spectroscopy (ROESY) experiments provided sufficient information to construct the complete structures of the new compounds $1-3$ and 11 known compounds $4,9,11,13$, $15,17,20,22,23,27$, and 31 . Structure analysis of the remaining compounds $5-8,10,12,14,16,18,19,21,24$ 26, $28-30$, and $32-38$ was performed useing one-dimensional NMR.

Sugikurojin A (1), $[\alpha]_{\mathrm{D}}^{25}+32.8^{\circ}$ was obtained as a colorless solid, and the molecular formula was established as $\mathrm{C}_{20} \mathrm{H}_{28} \mathrm{O}_{2}$, i.e., 16 mass units higher than that of 31, using high resolution (HR)-EI-MS, suggesting the presence of seven degrees of unsaturation. The absorption maxima at 281, 223, and $206 \mathrm{~nm}$ in the UV spectra suggested the presence of an aromatic ring. The ${ }^{13} \mathrm{C}-\mathrm{NMR}$ spectrum indicated<smiles>CC(C)c1cc2c(cc1O)[C@]1(C)CCCC(C)(C)[C@@]1(C)C=C2</smiles>

Sugikurojin A (1)

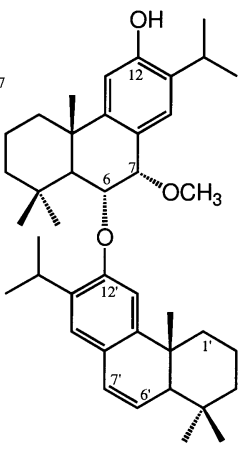

Sugikurojin B (2)

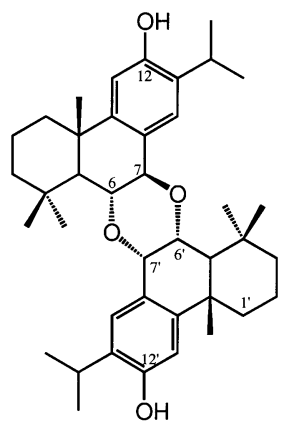

Sugikurojin C (3)<smiles>CC(C)c1cc2c(cc1O)C1(C)CCCC(C)(C)C1C1OC3c4cc(O)c(C(C)C)cc4C4C(OC3OC21)C(C)(C)CCCC4(C)C</smiles>

Formosaninol (4) 
the presence of four double bonds $[\delta 152.3$ (s, phenolic), 147.1 (s), 131.1 (s), 127.5 (d), 126.1 (d), 125.9 (s), 124.7 (d), 109.5 (d)] and an oxygenated carbon $\left[\delta 66.0(\mathrm{t})\right.$. Its ${ }^{1} \mathrm{H}-\mathrm{NMR}$ spectrum had signals for two angular methyl groups at $\delta 0.96$ $\left(\mathrm{H}_{3}-20\right)$ and $1.06\left(\mathrm{H}_{3}-18\right)$, one isopropyl group at $\delta 1.23$, $1.26\left(\mathrm{H}_{3}-16,-17\right)$, and $3.13(\mathrm{H}-15)$. Signals were also observed for hydroxymethyl protons at $\delta 3.73$ and $3.84\left(\mathrm{H}_{2}-\right.$ 19); an ABX system of vinyl protons at $\delta 5.98$ (H-6) and $6.49(\mathrm{H}-7)$ and a methine proton at $\delta 2.25(\mathrm{H}-5)$; signals for two aromatic protons at $\delta 6.59(\mathrm{H}-11)$ and $6.89(\mathrm{H}-14)$. The foregoing spectral data and literature survey provided evidence that $\mathbf{1}$ is a ferruginol derivative, with a double bond and an oxygenated methylene group $(\delta 66.0)$. The HMBC spectrum (Fig. 1) revealed that $\mathbf{1}$ had a hydroxy methylene at C-4 and a double bond at C-5. The nuclear Overhauser effect (NOE)s between $\mathrm{H}_{3}-20$ /hydroxymethyl and $\mathrm{H}-5$ /methyl at $\mathrm{C}$ 4 established the presence of a $\beta$ hydroxymethyl group at $\mathrm{C}$ 4. Hence, $\mathbf{1}$ is shown to be 19-hydroxy-6,7-dehydroferruginol.

Sugikurojin B (2), $[\alpha]_{\mathrm{D}}^{25}+148.2^{\circ}$ was obtained as a colorless solid. The FAB-MS of $\mathbf{2}$ gave pseudmolecular ions at $\mathrm{m} / \mathrm{z} 599[\mathrm{M}+\mathrm{H}]^{+}$and $637[\mathrm{M}+\mathrm{K}]^{+}$corresponding to a molecular formula of $\mathrm{C}_{41} \mathrm{H}_{58} \mathrm{O}_{3}$, which was confirmed by HR-EIMS, suggesting the presence of 13 degrees of unsaturation. The EI-MS displayed a molecular ion at $m / z 598(22 \%)$ with other significant fragment ions at $\mathrm{m} / \mathrm{z} 315$ (29\%), 284 (100\%), 283 (54\%), 213 (53\%), and 202 (80\%). The HR-EIMS exhibited fragment ions at $m / z 315$ (observed 315.2320; calcd. 315.2324 for $\mathrm{C}_{21} \mathrm{H}_{31} \mathrm{O}_{2}$ ) and 283 (observed 283.2052; calcd. 283.2062 for $\mathrm{C}_{20} \mathrm{H}_{27} \mathrm{O}$ ), indicating that compound 2 is composed of the constituent monomer- $1\left[\mathrm{C}_{21} \mathrm{H}_{31} \mathrm{O}_{2}\right]$ and -2 $\left[\mathrm{C}_{20} \mathrm{H}_{27} \mathrm{O}\right]$. The absorption bands at $3420,1605,1585,1425$, 12551090 , and $890 \mathrm{~cm}^{-1}$ in the IR spectrum and the $\lambda_{\max }$ at 290,231 , and $213 \mathrm{~nm}$ in the UV spectra suggested the presence of a hydroxyl group and an aromatic ring. Its ${ }^{1} \mathrm{H}-\mathrm{NMR}$ spectrum had signals for six angular methyl groups, two isopropyl groups, and one methoxy group. Signals were also observed for protons geminal to adjacent oxygen atoms at $\delta$ 3.98 (H-7) and $4.91(\mathrm{H}-6)$; an ABX system of two vinyl protons at $\delta 6.53\left(\mathrm{H}-6^{\prime}\right)$ and $5.90\left({\left.\mathrm{H}-7^{\prime}\right)}^{\prime}\right.$ and a methine proton at

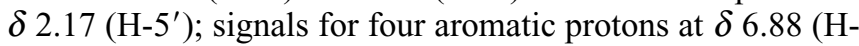
11), $6.94(\mathrm{H}-14), 7.04\left(\mathrm{H}-11^{\prime}\right)$, and $7.26\left(\mathrm{H}-14^{\prime}\right)$; and a signal for a phenolic hydroxyl proton at $\delta 4.77$ (H-12). Those NMR data suggested that one-half (constituent monomer-1) of the molecule is related to 6,7-dehydroferruginol $(\mathbf{3 1})^{36)}$ and the other (constituent monomer-1) related to $6 \alpha, 7 \alpha$-dihydroxyferruginol. ${ }^{9)}$ The gross structure of $\mathbf{2}$ was determined by analysis of NMR data including HMBC and ROESY experiments, and by referring to the NMR data for compound 31 and $6 \alpha, 7 \alpha$-dihydroxyferruginol. An HMBC experiment revealed long-range couplings from $\mathrm{H}-6$ at $\delta_{\mathrm{C}} 78.3$ to $\mathrm{C}-4$, $-5,-7$, and -8 ; from $\mathrm{H}-7$ at $\delta_{\mathrm{C}} 81.8$ to $\mathrm{C}-6,-8$, and -9 ; from HO- 12 at $\delta_{\mathrm{C}} 153.0$ to $\mathrm{C}-11,-12$, and -13 , indicating that the positions at C-6, C-7, and C-12 were oxygenated. Longrange couplings were also observed from $\mathrm{H}-7$ to the methoxy carbon and from the methoxy group to $\mathrm{C}-7$, indicating the methoxy group to be at C-7. Furthermore, the HMBC spectrum showed long-range coupling from H-6 to C-12', establishing the connectivity between the 6,7-dihydroxyferruginol and 6,7-dehydroferruginol. The stereochemistry of C-6 and C-7 were determined based on NOESY experiments and

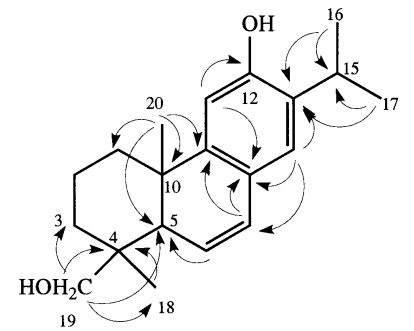

Fig. 1. HMBC Correlations for $\mathbf{1}$

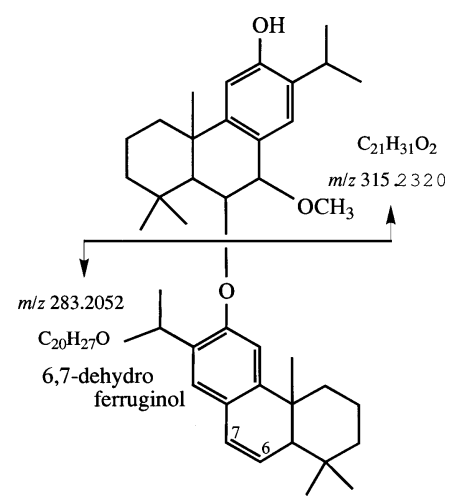

Fig. 2. Some Key HR-EI-MS Fragmentation Patterns of $\mathbf{2}$

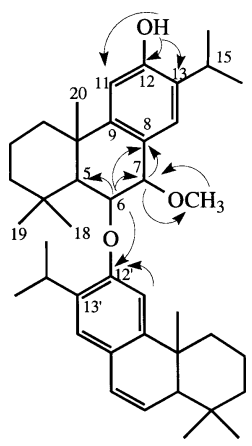

Fig. 3. Some Key HMBC of 2

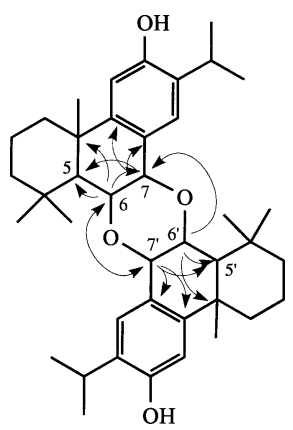

Fig. 4. Some Key HMBC of $\mathbf{3}$

coupling constants. The $\alpha-\mathrm{O}$ function at $\mathrm{C}-6$ could be assigned from the NOEs between $\mathrm{H}-6 / \mathrm{H}-19$ and -20 and the large $J_{\mathrm{H} 5-\mathrm{H} 6}$ coupling constant $(J=8.5 \mathrm{~Hz})$. The singlet proton signal at $\delta 3.98(\mathrm{H}-7)$ revealed the $\alpha$-configuration of the $\mathrm{O}$ function. Thus the structure of sugikurojin B was established as to be $\mathbf{2}$. Compound $\mathbf{2}$ was also detected in the hexane extract of $C$. japonica by HPLC. Therefore it is not a by-product in the methanol extraction. 
Sugikurojin $\mathrm{C}(\mathbf{3}),[\alpha]_{\mathrm{D}}^{25}-35.9^{\circ}$ was obtained as a colorless solid and assigned the molecular formula $\mathrm{C}_{40} \mathrm{H}_{56} \mathrm{O}_{4}$ on the basis of negative FAB-MS (M-H at $m / z$ 599), HR-EIMS $\left\{[\mathrm{M}]^{+}\right.$at $\left.m / z 600.4164\right\}$, and NMR data, corresponding to 13 degrees of unsaturation. The ${ }^{1} \mathrm{H}-\mathrm{NMR}$ spectrum (Table 1) of $\mathbf{3}$ exhibited two sets of signals typical of a derivative of ferruginol: an isopropyl group attached to a phenyl group; two para aromatic protons; and a typical $\mathrm{H} \beta-1$ proton at $\delta$ 2.17 (br d, $J=12.5 \mathrm{~Hz}$ ); as well as six singlet methyl groups. Signals were also observed for two pairs of three contiguous protons. Those of one pair were seen at $\delta 1.70(1 \mathrm{H}, \mathrm{d}$, $\left.J=11.5 \mathrm{~Hz}, \mathrm{H}-5^{\prime}\right), 4.08\left(1 \mathrm{H}, \mathrm{dd}, J=11.5,9.0 \mathrm{~Hz}, \mathrm{H}-6^{\prime}\right)$, and $4.56\left(1 \mathrm{H}, \mathrm{d}, J=9.0 \mathrm{~Hz}, \mathrm{H}-7^{\prime}\right)$, indicating $\beta-\mathrm{H}$ at the $\mathrm{C}-6^{\prime}$ position and $\alpha-\mathrm{H}$ at the $\mathrm{C}-7^{\prime}$ position. The other pair were seen

Table 1. NMR Data on Compounds $\mathbf{1}-\mathbf{4}$ in $\mathrm{CDCl}_{3}$

\begin{tabular}{|c|c|c|c|c|c|c|c|c|c|}
\hline \multirow[b]{2}{*}{ C no. } & \multicolumn{2}{|r|}{1} & \multicolumn{2}{|r|}{2} & \multicolumn{2}{|r|}{3} & \multirow[b]{2}{*}{ C no. } & \multicolumn{2}{|r|}{4} \\
\hline & $\begin{array}{c}{ }^{13} \mathrm{C} \delta \\
\text { (mult.) }\end{array}$ & ${ }^{1} \mathrm{H} \delta$ (mult. $J$ in $\mathrm{Hz}$ ) & $\begin{array}{l}{ }^{13} \mathrm{C} \delta \\
\text { (mult.) }\end{array}$ & ${ }^{1} \mathrm{H} \delta$ (mult. $J$ in $\mathrm{Hz}$ ) & $\begin{array}{l}{ }^{13} \mathrm{C} \delta \\
\text { (mult.) }\end{array}$ & ${ }^{1} \mathrm{H} \delta$ (mult. $J$ in $\mathrm{Hz}$ ) & & $\begin{array}{c}{ }^{13} \mathrm{C} \delta \\
\text { (mult.) }\end{array}$ & ${ }^{1} \mathrm{H} \delta$ (mult. $J$ in $\mathrm{Hz}$ ) \\
\hline \multirow[t]{2}{*}{1} & $35.9(\mathrm{t})$ & $\begin{array}{l}\mathrm{H}-\alpha 1.62 \text { (ddd, } 12.0 \\
\quad 12.0,5.0)\end{array}$ & $39.5(\mathrm{t})$ & $\begin{array}{l}\text { H-a } 1.58(\mathrm{ddd}, 12.5, \\
12.5,3.3)\end{array}$ & $38.8(t)$ & $\begin{array}{l}\text { H- } \alpha 1.51(\mathrm{ddd}, 12.5 \\
\quad 12.5,3.5)\end{array}$ & $1\left(1^{\prime}\right)$ & $38.6(t)$ & $\begin{array}{l}\text { H-a } 1.61(\mathrm{ddd}, 12.5, \\
12.5,3.3)\end{array}$ \\
\hline & & $\mathrm{H}-\beta 2.12(\mathrm{br} \mathrm{d}, 12.0)$ & & $\mathrm{H}-\beta 2.12(\mathrm{brd}, 12.5)$ & & $\mathrm{H}-\beta 2.17$ (br d, 12.5) & & & $\beta 2.11$ (brd, 12.5) \\
\hline \multirow[t]{2}{*}{2} & $18.7(\mathrm{t})$ & $\mathrm{H}-\alpha 1.69(\mathrm{~m})$ & $19.0^{a)}(\mathrm{t})$ & $\mathrm{H}-\alpha 1.66(\mathrm{~m})$ & $19.2(\mathrm{t})$ & $\mathrm{H}-\alpha 1.60(\mathrm{~m})$ & $2\left(2^{\prime}\right)$ & $18.9(\mathrm{t})$ & H-a $1.66(\mathrm{~m})$ \\
\hline & & $\begin{array}{c}\text { H- } \beta 1.74 \text { (ddddd, } 12.0, \\
12.0,12.0,5.0,5.0)\end{array}$ & & $\begin{array}{c}\mathrm{H}-\beta 1.86 \text { (ddddd, } 12.5 \text {, } \\
12.5,12.5,5.0,5.0)\end{array}$ & & $\begin{array}{c}\text { H- } \beta 1.71 \text { (ddddd, } 12.5 \\
12.5,12.5,3.5,3.5)\end{array}$ & & & $\begin{array}{c}\text { H- } \beta 1.75 \text { (ddddd, } 12.5 \text {, } \\
12.5,12.5,3.5,3.5)\end{array}$ \\
\hline \multirow[t]{2}{*}{3} & $35.1(\mathrm{t})$ & $\mathrm{H}-\alpha 1.07(\mathrm{~m})$ & $42.8(\mathrm{t})$ & $\mathrm{H}-\alpha 1.28(\mathrm{~m})$ & $42.7(\mathrm{t})$ & $\mathrm{H}-\alpha 1.21(\mathrm{~m})$ & $3\left(3^{\prime}\right)$ & $43.2(\mathrm{t})$ & $\begin{array}{l}\mathrm{H}-\alpha 1.35(\mathrm{ddd}, 12.5 \\
\quad 12.5,3.5)\end{array}$ \\
\hline & & $\mathrm{H}-\beta 1.87$ (br d, 14.0) & & $\mathrm{H}-\beta 1.87$ (br d, 12.5) & & $\mathrm{H}-\beta 1.43(\mathrm{brd}, 12.5)$ & & & H- $\beta 1.52($ br d, 12.5) \\
\hline 4 & $38.2(\mathrm{~s})$ & & $34.3(\mathrm{~s})$ & & $33.6(\mathrm{~s})$ & & $4\left(4^{\prime}\right)$ & $33.9(\mathrm{~s})$ & \\
\hline 5 & $50.9(d)$ & $2.25(\mathrm{dd}, 3.0,3.0)$ & $55.6(d)$ & $1.65(\mathrm{~d}, 8.5)$ & $45.5(d)$ & $2.13(\mathrm{~d}, 12.5)$ & $5\left(5^{\prime}\right)$ & $51.8(d)$ & $1.54(\mathrm{~d}, 9.5)$ \\
\hline 6 & $126.1(\mathrm{~d})$ & $5.98(\mathrm{dd}, 11.0,3.0)$ & $78.3(d)$ & $4.91(\mathrm{~d}, 8.5)$ & $73.2(\mathrm{~d})$ & $4.60(\mathrm{dd}, 12.5,4.5)$ & $6\left(6^{\prime}\right)$ & 73.1 (d) & $4.17(\mathrm{dd}, 9.5,7.5)$ \\
\hline 7 & $127.5(d)$ & $6.49(\mathrm{dd}, 11.0,3.0)$ & $81.8(d)$ & $3.98(\mathrm{~s})$ & $75.4(d)$ & $4.77(\mathrm{~d}, 4.5)$ & $7\left(7^{\prime}\right)$ & $71.8(\mathrm{~d})$ & $4.71(\mathrm{~d}, 7.5)$ \\
\hline 8 & $125.9(\mathrm{~s})$ & & $124.6(\mathrm{~s})$ & & $126.5(\mathrm{~s})$ & & $8\left(8^{\prime}\right)$ & $126.6(\mathrm{~s})$ & \\
\hline 9 & $147.1(\mathrm{~s})$ & & $150.8(\mathrm{~s})$ & & $147.5(\mathrm{~s})$ & & $9\left(9^{\prime}\right)$ & $147.2(\mathrm{~s})$ & \\
\hline 10 & $37.4(\mathrm{~s})$ & & $38.1(\mathrm{~s})$ & & $39.8(\mathrm{~s})$ & & $10\left(10^{\prime}\right)$ & $36.7(\mathrm{~s})$ & \\
\hline 11 & $109.5(\mathrm{~d})$ & $6.59(\mathrm{~s})$ & $110.4(d)$ & $6.88(\mathrm{~s})$ & $109.4(\mathrm{~d})$ & $6.60(\mathrm{~s})$ & $11\left(11^{\prime}\right)$ & $108.8(\mathrm{~d})$ & $6.62(\mathrm{~s})$ \\
\hline 12 & $152.3(\mathrm{~s})$ & & $153.0(\mathrm{~s})$ & $4.77(\mathrm{~s})$ & $152.5(\mathrm{~s})$ & & $12\left(12^{\prime}\right)$ & $151.8(\mathrm{~s})$ & \\
\hline 13 & $131.1(\mathrm{~s})$ & & $130.6(\mathrm{~s})$ & & $132.2(\mathrm{~s})$ & & $13\left(13^{\prime}\right)$ & $131.2(\mathrm{~s})$ & \\
\hline 14 & 124.7 (d) & $6.89(\mathrm{~s})$ & $129.5(d)$ & $6.94(\mathrm{~s})$ & $123.5(\mathrm{~d})$ & $7.26(\mathrm{~s})$ & $14\left(14^{\prime}\right)$ & $124.8(\mathrm{~d})$ & $7.46(\mathrm{~s})$ \\
\hline 15 & 26.7 (d) & $3.13(\mathrm{~m})$ & $25.5(\mathrm{~d})$ & $3.16(\mathrm{~m})$ & $26.4(\mathrm{~d})$ & $3.14(\mathrm{~m})$ & $15\left(15^{\prime}\right)$ & $26.9(\mathrm{~d})$ & $3.08(\mathrm{~m})$ \\
\hline 16 & $22.4(q)$ & $1.23(\mathrm{~d}, 7.0)$ & $23.2(q)$ & $1.05^{a)}(\mathrm{d}, 7.0)$ & $22.5(q)$ & $1.22(\mathrm{~d}, 7.0)$ & $16\left(16^{\prime}\right)$ & $22.3(q)$ & $1.20(\mathrm{~d}, 7.0)$ \\
\hline 17 & $22.8(q)$ & $1.26(\mathrm{~d}, 7.0)$ & $23.2(q)$ & $1.06^{a)}(\mathrm{d}, 7.0)$ & $22.7(q)$ & $1.27(\mathrm{~d}, 7.0)$ & $17\left(17^{\prime}\right)$ & $22.6(q)$ & $1.16(\mathrm{~d}, 7.0)$ \\
\hline 18 & $26.0(q)$ & $1.06(\mathrm{~s})$ & $35.1(q)$ & $0.93(\mathrm{~s})$ & $35.3(q)$ & $1.27(\mathrm{~s})$ & $18\left(18^{\prime}\right)$ & 35.7 (q) & $1.26(\mathrm{~s})$ \\
\hline 19 & $66.0(\mathrm{t})$ & $\begin{array}{l}3.73(\mathrm{~d}, 11.0) \\
3.84(\mathrm{~d}, 11.0)\end{array}$ & $22.9(q)$ & $1.17(\mathrm{~s})$ & $21.9(\mathrm{q})$ & $1.35(\mathrm{~s})$ & $19\left(19^{\prime}\right)$ & $22.9(q)$ & $1.14(\mathrm{~s})$ \\
\hline 20 & 20.7 (q) & $0.96(\mathrm{~s})$ & $24.7(q)$ & $1.39(\mathrm{~s})$ & $26.3(q)$ & $1.22(\mathrm{~s})$ & $20\left(20^{\prime}\right)$ & $22.9(q)$ & $1.21(\mathrm{~s})$ \\
\hline $\mathrm{O}-\mathrm{Me}$ & & & $55.1(q)$ & $3.16(\mathrm{~s})$ & & & & & \\
\hline \multirow[t]{2}{*}{$1^{\prime}$} & & & $36.3(\mathrm{t})$ & H-ax $1.73(\mathrm{~m})$ & $38.6(\mathrm{t})$ & $\begin{array}{l}\text { H-ax } 1.40(\mathrm{ddd}, 12.0, \\
12.0,4.0)\end{array}$ & & & \\
\hline & & & & H-e $2.24(\mathrm{br} \mathrm{d}, 12.5)$ & & H-e 2.17 (br d, 12.0) & & & \\
\hline \multirow[t]{2}{*}{$2^{\prime}$} & & & $19.1^{a)}(\mathrm{t})$ & $\begin{array}{l}\text { H-ax } 1.79 \text { (ddddd, } \\
12.5,12.5,12.5 \\
3.5,3.5)\end{array}$ & $18.9(\mathrm{t})$ & $\begin{array}{l}\text { H-e } 1.74 \text { (ddddd, } 12.0 \text {, } \\
12.0,12.0,4.0,4.0)\end{array}$ & & & \\
\hline & & & & H-e $1.74(\mathrm{~m})$ & & H-ax $1.60(\mathrm{~m})$ & & & \\
\hline \multirow[t]{2}{*}{$3^{\prime}$} & & & $41.1(\mathrm{t})$ & H-ax $1.28(\mathrm{~m})$ & $42.7(\mathrm{t})$ & H-ax $1.21(\mathrm{~m})$ & & & \\
\hline & & & & H-e 1.54 (br d, 12.5) & & H-e 1.43 (br d, 14.0) & & & \\
\hline $4^{\prime}$ & & & $32.9(\mathrm{~s})$ & & $33.2(\mathrm{~s})$ & & & & \\
\hline $5^{\prime}$ & & & $51.0(\mathrm{~d})$ & $2.17(\mathrm{dd}, 3.0,2.5)$ & $52.2(\mathrm{~d})$ & $1.70(\mathrm{~d}, 11.5)$ & & & \\
\hline $6^{\prime}$ & & & $127.2(\mathrm{~d})$ & $6.53(\mathrm{dd}, 9.5,3.0)$ & $77.7(\mathrm{~d})$ & $4.08(\mathrm{dd}, 11.5,9.0)$ & & & \\
\hline $7^{\prime}$ & & & $127.4(d)$ & $5.90(\mathrm{dd}, 9.5,2.5)$ & $74.1(\mathrm{~d})$ & $4.56(\mathrm{~d}, 9.0)$ & & & \\
\hline $8^{\prime}$ & & & $125.5(\mathrm{~s})$ & & $126.0(\mathrm{~s})$ & & & & \\
\hline $9^{\prime}$ & & & $147.0(\mathrm{~s})$ & & $147.2(\mathrm{~s})$ & & & & \\
\hline $10^{\prime}$ & & & $37.9(\mathrm{~s})$ & & $40.9(\mathrm{~s})$ & & & & \\
\hline $11^{\prime}$ & & & $106.6(d)$ & $7.04(\mathrm{~s})$ & $111.2(\mathrm{~d})$ & $6.61(\mathrm{~s})$ & & & \\
\hline $12^{\prime}$ & & & $152.9(\mathrm{~s})$ & & $152.4(\mathrm{~s})$ & & & & \\
\hline $13^{\prime}$ & & & $134.8(\mathrm{~s})$ & & $132.0(\mathrm{~s})$ & & & & \\
\hline $14^{\prime}$ & & & $124.5(\mathrm{~d})$ & $7.26(\mathrm{~s})$ & $123.5(\mathrm{~d})$ & $7.45(\mathrm{~s})$ & & & \\
\hline $15^{\prime}$ & & & $26.5(d)$ & $3.16(\mathrm{~m})$ & $26.9(\mathrm{~d})$ & $3.14(\mathrm{~m})$ & & & \\
\hline $16^{\prime}$ & & & $22.5(q)$ & $1.19(\mathrm{~d}, 7.0)$ & $22.5(q)$ & $1.22(\mathrm{~d}, 7.0)$ & & & \\
\hline $17^{\prime}$ & & & $22.6(q)$ & $1.22(\mathrm{~d}, 7.0)$ & $22.6(q)$ & $1.25(\mathrm{~d}, 7.0)$ & & & \\
\hline $18^{\prime}$ & & & $32.6(q)$ & $0.99(\mathrm{~s})$ & $35.9(q)$ & $1.10(\mathrm{~s})$ & & & \\
\hline $19^{\prime}$ & & & $22.7(q)$ & $1.07(\mathrm{~s})$ & $22.4(q)$ & $1.27(\mathrm{~s})$ & & & \\
\hline $20^{\prime}$ & & & $20.4(q)$ & $1.12(\mathrm{~s})$ & $27.5(q)$ & $1.35(\mathrm{~s})$ & & & \\
\hline
\end{tabular}


at $\delta 2.13(1 \mathrm{H}, \mathrm{d}, J=12.5 \mathrm{~Hz}, \mathrm{H}-5), 4.60(1 \mathrm{H}, \mathrm{dd}, J=12.5$, $4.5 \mathrm{~Hz}, \mathrm{H}-6)$, and $4.77(1 \mathrm{H}, \mathrm{d}, J=4.5 \mathrm{~Hz}, \mathrm{H}-7)$, indicating $\beta$ $\mathrm{H}$ at the $\mathrm{C}-6$ position and $\beta-\mathrm{H}$ at the $\mathrm{C}-7$ position. Hence the structure of sugikurojin $\mathrm{C}$ can be either as drawn in formula 3 (6-O-7' and 7-O-6' linkage) or its isomer (6-O-6' and 7-O$7^{\prime}$ linkage). The $\mathrm{HMBC}$ experiment revealed long-range couplings from the $\mathrm{H}-6$ proton to $\mathrm{C}-7^{\prime}$ carbon, from the $\mathrm{H}-7$ proton to $\mathrm{C}-6^{\prime}$ carbon, and from the $\mathrm{H}-6^{\prime}$ proton to $\mathrm{C}-7$ carbon, suggesting that 3 has a $6-O-7^{\prime}$ and $7-O-6^{\prime}$ linkages. Hence the structure of sugikurojin $\mathrm{C}$ was established to be 3 .

Compound $4,[\alpha]_{\mathrm{D}}^{25}+36.3^{\circ}$ had the same molecular structure, $\mathrm{C}_{40} \mathrm{H}_{56} \mathrm{O}_{4}$ (HR-EI-MS, $m / z$ 600.4163) as 3. Its NMR spectra revealed only 20 carbon signals and the proton signals due to a dioxygenated ferruginol, suggesting that $\mathbf{4}$ is a dimeric dimer of dioxygenated ferruginol. By analysis of NMR data including ${ }^{1} \mathrm{H}-{ }^{1} \mathrm{H}$ COSY, ${ }^{1} \mathrm{H}-{ }^{13} \mathrm{C}$ COSY, HMBC and ROESY experiment, the structure of $\mathbf{4}$ was determined to be $[4 \mathrm{~b} S-(4 \mathrm{~b} \alpha, 8 \mathrm{a} \beta, 8 \mathrm{~b} \alpha, 9 \mathrm{a} \beta, 13 \mathrm{~b} \alpha, 17 \mathrm{a} \beta, 17 \mathrm{~b} \beta, 18 \mathrm{a} \beta)]-$ $4 \mathrm{~b}, 5,6,7,8,8 \mathrm{a}, 8 \mathrm{~b}, 9 \mathrm{a}, 13 \mathrm{~b}, 14,15,16,17,17 \mathrm{a}, 17 \mathrm{~b}, 18 \mathrm{a}-\mathrm{hexade}-$ cahydro-4b, 8, 8, 13b, 17, 17-hexame thyl-2,11-bis( 1 methylethyl)-diphenanthro[9,10-b:9',10'-e][1,4]dioxin-3,12diol, or formosaninol, which was isolated from the heartwood of Juniperus formosa HAY. var. concolor by Kuo et al. ${ }^{10)}$ However, the assignments of C-4, $-6,-7$ and -18 in the literature are incorrect. Here we reported the complete NMR data of formosaninol on the basis of 2D-NMR.

\section{Experimental}

General Experimental Procedures Optical rotations were recorded on a JASCO DIP-140 digital polarimeter. IR spectra were measured on a JASCO FT/IR-5300 instrument. UV spectra were recorded with a Shimadzu UV-6000 spectrophotometer. NMR spectra were recorded on a Varian UNITY 600 spectrometer in $\mathrm{CDCl}_{3}$ solution using tetramethylsilane (TMS) as an internal standard. NMR experiments included ${ }^{1} \mathrm{H}-{ }^{1} \mathrm{H}$ COSY, HMQC, HMBC, and ROESY. Coupling constants ( $J$ values) are given in Hertz (Hz). HR-EI-MS was measured on a JEOL JMS-PX303 mass spectrometer. Kieselgel 60 (230 - 400 mesh, Merck) was used for column chromatography, and silica gel 60F-254 (Merck) for TLC. HPLC was carried out on a JASCO-PU 1580 instrument using a COSUMOSIL C18 P-MS $(4.6 \times 150$, $20 \times 250 \mathrm{~mm}$ ) column.

Plant Material The black heartwood of C. japonica trees aged 70 to 80 years from Kaifu, Tokushima, was collected in October 2000. The voucher specimen (3001) is deposited in the Herbarium of the Department of Pharmacognosy, Tokushima Bunri University, Tokushima, Japan.

Extraction and Isolation The chips $(3.92 \mathrm{~kg})$ of $C$. japonica heartwood were exhaustively extracted with $\mathrm{MeOH}$ at room temperature for 4 weeks. The methanol extract was evaporated under a vacuum to yield a brown residue $\left(296 \mathrm{~g}\right.$ ) that was partitioned between $\mathrm{H}_{2} \mathrm{O}$ and EtOAc. Repeated separation of the aliquot $(120 \mathrm{~g})$ of the EtOAc-soluble portion $(240 \mathrm{~g})$ by chromatography over ordinary-phase silica gel (using increasing concentration of diisopropyl ether in hexane as eluent) and reverse-phase HPLC (70-90\% $\mathrm{MeOH})$ furnished sugikurojins A $(\mathbf{1}, 9 \mathrm{mg}), \mathrm{B}(\mathbf{2}, 60 \mathrm{mg})$, and C $(\mathbf{3}, 10 \mathrm{mg})$, formosaninol $(4,9 \mathrm{mg}), \alpha$-muurolene $(5,20 \mathrm{mg}), \delta$-cadinene $(6,25 \mathrm{mg})$, calamenene $(7,20 \mathrm{mg})$, T-cadinol $(\mathbf{8}, 8 \mathrm{mg})$, cubenol $(\mathbf{9}, 31 \mathrm{mg})$, epi-cubenol $(\mathbf{1 0}, 70 \mathrm{mg})$, cubebol $(\mathbf{1 1}, 50 \mathrm{mg})$, epi-cubebol $(\mathbf{1 2}, 11 \mathrm{mg})$, gleenol (13, $6.5 \mathrm{mg}), \beta$-elemol $(\mathbf{1 4}, 11 \mathrm{mg})$, cryptomerione $(\mathbf{1 5}, 140 \mathrm{mg})$, 4-hydroxy7(14), 10-bisaboladien-1-one (16, $8 \mathrm{mg})$, 4-hydroxy-2,7(14),10-bisabolatrien1-one (17, $10 \mathrm{mg}),-7(14), 10$-bisaboladien-1,4-diol (18, $8 \mathrm{mg})$, cryptomeridiol $(\mathbf{1 9}, 24 \mathrm{mg})$, sandracopimaradiene $(\mathbf{2 0}, 6 \mathrm{mg})$, sandaracopimarinal (21, $20 \mathrm{mg})$, sandaracopimarinol $(\mathbf{2 2}, 30 \mathrm{mg})$, sandaracopimaric acid $(\mathbf{2 3}, 22 \mathrm{mg})$, 8(14),15-pimaradiene-3-ol (24, $7 \mathrm{mg})$, 18-nor-8(14),15-isopimaradien-4-ol $(25,15 \mathrm{mg})$, sandaracopimarinol acetate $(\mathbf{2 6}, 6 \mathrm{mg}), 16$-phyllocladanol $(\mathbf{2 7}$, $30 \mathrm{mg}), \mathbf{7}, 13$-abietadiene $(\mathbf{2 8}, 15 \mathrm{mg})$, abietatiene $(\mathbf{2 9}, 18 \mathrm{mg})$, ferruginol (30,650 mg), dehydroferruginol (31, $185 \mathrm{mg})$, sugiol (32, $110 \mathrm{mg})$, hinokiol (33, 7 mg), 19-hydroxyferruginol (34, 7 mg), 6,7-secoferruginol-6,7-dial $(35,15 \mathrm{mg})$, sequiein C (36, $40 \mathrm{mg})$, sugirecinol $(\mathbf{3 7}, 10 \mathrm{mg})$, agatharecinol (38, $40 \mathrm{mg})$, and 4-hydroxybenzaldehyde $(\mathbf{3 9}, 22 \mathrm{mg})$ were identified by comparison with published data.
Sugikurojin A (1): A colorless solid; $[\alpha]_{\mathrm{D}}^{25}+32.8^{\circ}\left(c=0.39, \mathrm{CHCl}_{3}\right)$; FTIR (dry film) $3400 \mathrm{~cm}^{-1}$; UV (MeOH) $\lambda_{\max }(\log \varepsilon) \mathrm{nm} 206$ (4.04), 223 (3.81), 281 (3.60); selected NOESY data, $1 \alpha / 5,3 \alpha / 5 \alpha, 5 / 18,7 / 14,2 \beta / 19$, $2 / 20,19 / 20,15 / 16,15 / 17,16 / 17$; HR-EI-MS $\mathrm{m} / z 300.2083$ (calcd. for $\left.\mathrm{C}_{20} \mathrm{H}_{28} \mathrm{O}_{2}, 236.2140\right) .{ }^{1} \mathrm{H}$ - and ${ }^{13} \mathrm{C}$-NMR see Table 1 .

Sugikurojin B (2): A colorless solid; $[\alpha]_{\mathrm{D}}^{25}+148.2^{\circ}\left(c=1.57, \mathrm{CHCl}_{3}\right)$; FTIR (dry film) 3420, 1605, 1585, 1425, $12551090,890 \mathrm{~cm}^{-1}$; UV (MeOH) $\lambda_{\max }(\log \varepsilon) \mathrm{nm} 213$ (3.92), 231 (3.66), 290 (3.32); ${ }^{1} \mathrm{H}-,{ }^{13} \mathrm{C}-\mathrm{NMR}$, and HMBC see Table 2; selected NOESY data, $1 \alpha / 3 \alpha, 1 \alpha / 5 \alpha, 1 \beta / 11,3 \alpha / 5 \alpha$, 5/18, 6/20, 6/11', 7/14, 7/OMe, 7/11', 11/HO-12, HO-12/15, 14/15, 14/16, 14/17, 16/17, 18/19, 19/20, 1'ax/3'ax, 1'ax/5'ax, 1' eq/11', 3'ax/5'ax, $5^{\prime}$ ax $/ 18^{\prime}, 6^{\prime} / 7^{\prime}, 7^{\prime} / 14^{\prime}, 14^{\prime} / 15^{\prime}, 14^{\prime} / 16^{\prime}, 14^{\prime} / 17^{\prime}, 16^{\prime} / 17^{\prime}, 18^{\prime} / 19^{\prime}, 19^{\prime} / 20^{\prime}$; EI-MS $m / z: 598\left(\mathrm{M}^{+}, 22\right), 566(26), 315$ (29), $285(35), 284$ (100), $283(54)$, 213 (53), 202 (80), 199 (41), 159 (23); positive FAB-MS $m / z$ : $599(\mathrm{M}+\mathrm{H})^{+}$, $637(\mathrm{M}+\mathrm{K})^{+}$; HR-EI-MS m/z: 598.4381 (calcd. for $\mathrm{C}_{41} \mathrm{H}_{58} \mathrm{O}_{3}, 598.4386$ ), m/z 315 (observed 315.2320; calcd. 315.2324 for $\mathrm{C}_{21} \mathrm{H}_{31} \mathrm{O}_{2}$ ) and 283 (observed 283.2052; calcd. 283.2062 for $\mathrm{C}_{20} \mathrm{H}_{27} \mathrm{O}$ ). ${ }^{1} \mathrm{H}$ - and ${ }^{13} \mathrm{C}-\mathrm{NMR}$, see Table 1.

Sugikurojin C (3): A colorless solid; $[\alpha]_{\mathrm{D}}^{20}-35.9^{\circ}\left(c=0.62, \mathrm{CHCl}_{3}\right)$ FT-IR (dry film) $3410 \mathrm{~cm}^{-1}$; UV (MeOH) $\lambda_{\max }(\log \varepsilon) \mathrm{nm} 209$ (4.47), 225 (4.17), 279 (3.66); EI-MS m/z: 600 (M+1, 86), 300 (65), 284 (100), 241(39), 202 (29), 199 (28), 185(20), 159 (18); negative FAB-MS m/z $599(\mathrm{M}-\mathrm{H})^{+}$; HREI-MS $m / z 600.4164$ (calcd. for $\mathrm{C}_{40} \mathrm{H}_{56} \mathrm{O}_{4}, 600.4178$ ), $\mathrm{m} / \mathrm{z} 300$ (observed 300.2099; calcd. 300.2090 for $\mathrm{C}_{20} \mathrm{H}_{28} \mathrm{O}_{2}$ ); selected NOESY data, $1 \alpha / 3 \alpha$, $1 \alpha / 5 \alpha, 1 \beta / 11,3 \alpha / 5 \alpha, 5 / 7,5 / 18,6 / 7^{\prime}, 6 / 19,6 / 20,7 / 5^{\prime}, 14 / 15,14 / 16,14 / 17$, $14 / 18^{\prime}, 16 / 17,18 / 19,19 / 20,1^{\prime} \alpha / 3^{\prime} \alpha, 1^{\prime} \alpha / 5^{\prime} \alpha, 1^{\prime} \beta / 11,3^{\prime} \alpha / 5^{\prime} \alpha, 5^{\prime} / 18^{\prime}$, $6^{\prime} / 7^{\prime}, 6^{\prime} / 19^{\prime}, 6^{\prime} / 20^{\prime}, 7^{\prime} / 14^{\prime}, 14^{\prime} / 15^{\prime}, 14^{\prime} / 16^{\prime}, 14^{\prime} / 17^{\prime}, 14^{\prime} / 18^{\prime}, 16^{\prime} / 17^{\prime}$, $18^{\prime} / 19^{\prime}, 19^{\prime} / 20^{\prime}$. ${ }^{1} \mathrm{H}$ - and ${ }^{13} \mathrm{C}-\mathrm{NMR}$, see Table 1 .

Formosaminol (4): A colorless solid; $[\alpha]_{\mathrm{D}}^{20}+36.3^{\circ}\left(c=0.48, \mathrm{CHCl}_{3}\right)$; FTIR (dry film) $3420 \mathrm{~cm}^{-1}$; UV (MeOH) $\lambda_{\max }(\log \varepsilon) \mathrm{nm} 208$ (4.46), 233 (3.98), 279 (3.61); EI-MS m/z: $600\left(\mathrm{M}^{+}, 100\right), 300$ (17), 314 (10), 284 (60), 283 (31), 202 (27), 199 (12), 159 (12) negative FAB-MS m/z $599(\mathrm{M}-\mathrm{H})^{+}$; HR-EI-MS $m / z 600.4163$ (calcd. for $\mathrm{C}_{40} \mathrm{H}_{56} \mathrm{O}_{4}, 600.4178$ ), $\mathrm{m} / \mathrm{z} 300$ (observed 300.2061; calcd. 300.2090 for $\mathrm{C}_{20} \mathrm{H}_{28} \mathrm{O}_{2}$ ); selected NOESY data, $1 \alpha / 3 \alpha, 1 \alpha / 5 \alpha, 1 \beta / 11,3 \alpha / 5 \alpha, 5 / 18,6 / 7,6 / 19,6 / 20,7 / 14,14 / 15,14 / 16$, $14 / 17,14 / 18^{\prime}, 16 / 17,18 / 19,19 / 20,1^{\prime} \alpha / 3^{\prime} \alpha, 1^{\prime} \alpha / 5^{\prime} \alpha, 1^{\prime} \beta / 11^{\prime}, 3^{\prime} \alpha / 5^{\prime} \alpha$, $5^{\prime} / 18^{\prime}, 6^{\prime} / 7^{\prime}, 6^{\prime} / 7^{\prime}, 6^{\prime} / 20^{\prime}, 7^{\prime} / 14^{\prime}, 14^{\prime} / 15^{\prime}, 14^{\prime} / 16^{\prime}, 14^{\prime} / 17^{\prime}, 14^{\prime} / 18,16^{\prime} / 17^{\prime}$, $18^{\prime} / 19^{\prime}, 19^{\prime} / 20^{\prime}$. ${ }^{1} \mathrm{H}$ - and ${ }^{13} \mathrm{C}-\mathrm{NMR}$, see Table 1.

Acknowledgment This work was supported by Japan Science and Technology Corporation.

\section{References and Notes}

1) Shieh B., Iizuka Y., Matsubara Y., Agric. Biol. Chem., 45, 1493-1495 (1981).

2) Nagahama S., Tazaki M., Mokuzai Gakkaishi, 39, 1077-1083 (1993).

3) Su W.-C., Fang J.-M., Cheng Y.-S., Phytochemistry, 35, 1279-1284 (1994) and references cited therein.

4) Nagahama S., Tazaki M., Nomura H., Nishimura K., Tajima M., Iwashita Y., Mokuzai Gakkaishi, 42, 1127-1133 (1996).

5) Ashitani T., Iwaoka T., Nagahama S., Nat. Prod. Lett., 13, 169-170 (1999).

6) Nagahama S., Tukamoto T., Torii N., Sonoda T., Yamanobe T., Mokuzai Gakkaishi, 47, 487-492 (2001).

7) Tebayashi S., Horiike M., Biosci. Biotechnol. Biochem., 65, 14341437 (2001).

8) Kofujita H., Fujino Y., Sasaki T., Hasebe M., Ota M., Suzuki K., Mokuzai Gakkaishi, 47, 479-486 (2001).

9) Nagahama S., Fujii H., Sonoda T., Sasaki M., Mokuzai Gakkaishi, 48, 384-386 (2002)

10) Kuo Y.-H., Yu M.-T., Chem. Pharm. Bull., 44, 1431-1435 (1996).

11) Beecham C. M., Djerassi C., Tetrahedron, 34, 2503-2508 (1978).

12) Randriamiharisoa R., Gaydou E. M., Faure R., Bianchini J. P., Magn. Reson., 24, 275-276 (1968).

13) Croft K. D., Ghisalberti E. L., Hocart C. H., Jefferies P. R., Raston C. L., White A. H. J. Chem. Soc. Perkin Trans. 1, 1978, 1267-1270 (1978).

14) Melching S., Buelow N., Wihstutz K., Jung S., Koenig W. A., Phytochemistry, 44, 1291-1296 (1997).

15) Labbe C., Castillo M., Connolly J. D., Phytochemistry, 34, 441-444 (1993).

16) Ohta Y., Hirose Y. Tetrahedron Lett., 1967, 2073-2075 (1967).

17) Connolly J. D., Phillips W. R., Huneck S., Phytochemistry, 21, 233- 
234 (1982)

18) De Rosa De Giulio A., Iodice C., Zavodink N., Phytochemistry, 37, 1327-1330 (1994).

19) Barrero A. F., Sanchez J. F., Oltra J. E., Altarejos J., Ferrol N., Barragan A., Phytochemistry, 30, 1551-1554 (1991).

20) Su W.-C., Fang J.-M., Cheng Y.-S., Phytochemistry, 39, 603-607 (1995).

21) Gutierrez A. B., Herz W., Phytochemistry, 27, 3871-3874 (1988).

22) The naming of compound $\mathbf{1 4}$ followed. Ref. 18. Nagahama S., Tazaki M., Nomura H., Nishimura K., Tajima M., Iwashita Y., Mokuzai Gakkaishi, 42, 1127-1133 (1996).

23) The naming of compound $\mathbf{1 5}$ followed. Ref. 19. Nagahama S., Tazaki, M., Mokuzai Gakkaishi, 39, 1077-1083 (1993).

24) Nakashima H., Miyano N., Sawabe Y., Takatuka T., Sen'i Gakkaishi, 58, 129-134 (2002).

25) Lin T.-C., Fang J.-M., Cheng Y.-S., Phytochemistry, 51, 793-801 (1999).

26) Guajardo T. E. M., Lopez E. G., Reyes P. A., Sanchez H., Honecker F., Achenbach H., Phytochemistry, 45, 387-390 (1997).

27) Rezzi S., Bighelli A., Castola V. C., J. App. Spectroscopy, 56, 312 317 (2002).

28) Wenlert E., Buckwalter B. E., J. Am. Chem. Soc., 94, 4367-4369
(1972).

29) Sakar M. K., San Feliciano A., Fitoterapia, 65, 304-306 (1994).

30) Ansell S. M., Pegal K. H., Taylor D. A. H., Phytochemistry, 32, $953-$ 959 (1993).

31) Lee C.-K., Fang J.-M., Cheng Y.-S., Phytochemistry, 39, 391-394 (1995).

32) Patra A., Mitra A. K., Mitra S. R., Kirtaniya C. L., Adityachaudhury N., Org. Magn. Reson., 14, 58-60 (1980).

33) San Feliciano A., Miguel del Corral J. M., Gordaliza M., Salinero M. A., Magn. Reson. Chem., 31, 841-844 (1993).

34) Miguel del Corral J. M., Gordaliza M., Salinero M. A., San Feliciano A., Magn. Reson. Chem., 32, 703 (1994).

35) Bredenberg J. B.-S., Acta Chem. Scand., 11, 98-100 (1957).

36) Lin Y. T., Kuo Y. H, Chang B. H., J. Chim. Chem. Soc. (Taipei), 22, 331-334 (1975).

37) Zhao Q.-S., Tian J., Yue J.-M., Chen S.-N., Lin Z.-W., Sun H.-D., Phytochemistry, 48, 1025-1029 (1998).

38) Fang J., Jan S., Cheng Y. J., Chem. Res. Synop., 1986, 350-351 (1986).

39) Yoshida S., Morita Y., Narita K., Okabe T., Tennen Yuki Kagabutu Toronkai, 40th, 1998, pp. 311-315. 